\title{
Buoyancy of Topics on Communications Software and Systems
}

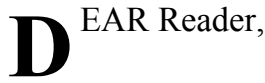

On behalf of the Editorial Board and the Steering Committee, welcome to the first issue of the JOURNAL OF COMMUNiCATIONS SOFTWARE AND SYSTEMS. The journal is published by the Croatian Communications and Information Society (CCIS) in cooperation with FESB, University of Split. The JOURNAL OF COMMUNICATIONS SOFTWARE AND SYSTEMS is endorsed by the IEEE Technical Committee on Communications Software of the IEEE Communications Society (ComSoc).

This journal is focused on high-quality, internationally reviewed papers that advance the state-of-the-art applications in communications software engineering and communications systems, particularly including new technologies, methods and algorithms, as well as reports on experimental analysis of performances and prototypes. Editorial Board is particularly interested in research results that contribute to the scientific understanding of the impacts that communication software engineering can have on systems and service management, quality control and reliability issues. It is our aim to provide the reader with the opportunity of benefiting from experience, problems and solutions described.

The topics covered by this journal include various aspects of communications software and systems in wired and wireless communications including multimedia technologies that have a significant impact on enormous technical and commercial opportunities in the ICT sector. More specifically, the JOURNAL OF COMMUNICATIONS SOFTWARE AND SYSTEMS will cover (but not be limited) to the following: Theoretical Aspects of Communications Software and Systems (e.g. Data Compression and Source Coding Issues); Communications Software Design Cycle and related techniques (e.g. UML); Software for Network Planning, Design, Operations and Management (e.g. Strategy Planning and Management in Telecommunications); Communications Software Quality Issues; Software and Systems Issues in Communication Protocols and Architectures (including Mobile and Wireless Networks); Artificial Intelligence in Communications Software (Knowledge-based Systems, Intelligent Agents, Human Systems and Human-Computer Interaction; Soft Communication and Information Systems, Pattern Recognition Methods, Robotics and Control Systems; Decision Support Systems); Multimedia and Virtual Reality in Communications Systems; Quality of Service (QoS) in Communications Systems; System's Security Issues in Optical, Photonic and Satellite Systems; Information Infrastructure and Security; Software and Methods for Signal Processing; Emerging Network Systems and Services (Home Networks, Powerline/ Wireless Access, Sensor Networks, Electromagnetic Compatibility Issues, Consumer Device Communications,
Communications Methods in Biomedicine); Software in Enterprise Networking; Middleware in Grid Computing Systems; Research and Education Methodology in the area of Communications Software and Systems.

Authors are invited to submit their high-quality papers presenting the original results in all the above mentioned areas of communications software and systems. Submission of the original manuscript to the Journal will be taken to mean that it represents the original work neither previously published, nor being considered elsewhere for publication. Revised and extended versions of papers printed in conference/symposium/ workshop proceedings may also be submitted to the journal with an appropriate cover note.

JCOMSS is an all-electronic processing journal, published quarterly, typically offering a 6 months' window for final decision about publishing well prepared regular papers, accepted after one round of reviews. Each additional review round will extend the window for up to 3 months approximately. This relatively rapid reviewing process is possible owing to highly automated electronic reviewing process, but also owing to the concerted effort of the editorial board members, reviewers, and authors. We have practiced such concerted action in our past work and we strongly believe that this working atmosphere will continue in future.

Finally, I wish to acknowledge the efforts of our Editors-inChief, Prof. Algirdas Pakstas of the London Metropolitan University, Prof. Dinko Begusic of the University of Split, and Prof. Mladen Kos of the University of Zagreb. I would also like to express deep gratitude to Dr.sc. Roberto Saracco of the Telecom Italy and Dr.sc. Naohisa Ohta of the Research Institute for Digital Media \& Content, Japan. They have encouraged the foundation of this journal as a step towards promoting further collaboration and activities of the IEEE ComSoc Sister Societies.

NiKola A. RozIC

Chair, Steering Committee rozic@fesb.hr 